\title{
Examining Models of Nondominated Decoy Effects across J udgment and Choice
}

\author{
J onathan C. Pettibone and Douglas H. Wedell
}

University of South Carolina

\begin{abstract}
Three experiments explored cognitive models of inferior, compromise, and phantom decoy effects in both judgment and choice. Partici pants made judgments of attractiveness, justifiability, and evaluation anxiety associated with each alternative in the set, along with judgments of the attractiveness of each alternative's dimensional values. In another session, they also chose the alternative they most preferred. Results were analyzed in terms of the degree to which decoy effects reflected shifts in dimensional values or reflected emergent values based on relationships with other alternatives in the set. Both emergent-value and value-shift models of inferior decoy effects were supported, but only the emergent-value model of compromise decoy effects was supported. Results for the phantom decoy indicated that this effect was choice-based and did not occur in judgment. Thus, although decoy effects were largely similar in choice and judgment, they also differed in important ways. (2000 Academic Press
\end{abstract}

When making choices, certain alternatives may lure the unsuspecting decision maker into choosing onealternative over another. These alternatives, such as those described by the attraction and compromise effects (Huber, Payne, \& Puto, 1982; Huber \& Puto, 1983), are rarely chosen and yet strongly influence the choice process. We refer to such alternatives as decoys, for, despite their differences, their presence serves to increase preference for another alternative in the choice set (Wedell \& Pettibone, 1996).

Decoy effects provide insights into the contextual nature of choice and as

This research was supported by NSF Grant SBR-9319520. Experiments 1 and 2 were based on work completed as part of the first author's master's research.

Address correspondence and reprint requests to J onathan C. Pettibone, Department of Psychology, University of South Carolina, Columbia, SC 29208. E-mail: Pettibone@sc.edu. 


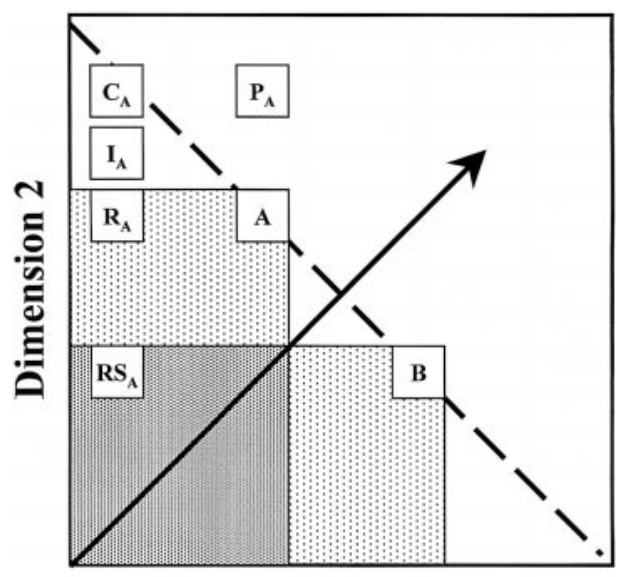

Dimension 1

FIG. 1. Placements of compromise $\left(C_{A}\right)$, inferior $\left(I_{A}\right)$, and phantom $\left(P_{A}\right)$ decoys, all targeting alternative $A$ over $B$, described on two dimensions. Regions of dominance are depicted as shaded boxes. For comparison, range $\left(R_{A}\right)$ and range symmetrical $\left(R_{A}\right)$ decoys are also shown.

such have been widely studied. These effects are robust and have been replicated with many different types of decoys and stimulus materials, demonstrating violations of normative choice principles (Ariely \& Wallsten, 1995; Dhar \& Glazer, 1996; Huber, Payne, \& Puto, 1982; Huber \& Puto, 1983; Simonson, 1989; Wedell, 1991; Wedell \& Pettibone, 1996). They have also been found to affect real-world consumer purchasing, such as decisions to purchase products in supermarket settings (Doyle, O'Connor, Reynolds, \& Bottomley, 1999). The study of decoy effects is thus directly applicable to both theoretical and applied interests.

Across multiple studies, two general classes of decoys have emerged which differ in the relationship between the decoy and the rest of the set. Dominated decoys, such as asymmetrically dominated (Huber et al., 1982) and symmetrically dominated decoys (Wedell, 1991), share the property of being dominated by one or more alternatives. Being dominated refers to having at least one feature that is clearly worse than those of a competing alternative and no features that are better. In contrast, nondominated decoys increase preference for the targeted alternative without being dominated. These decoys tend to have similar but smaller effects compared to asymmetrically dominated decoys. This class of decoys includes compromise (Simonson, 1989), inferior (Huber \& Puto, 1983), and phantom decoys (Pratkanis \& Farquhar, 1992).

Given the structural differences between these two classes of decoys, we would expect the processes governing their effects to differ. One goal of the present research was to compare and contrast cognitive models of the different nondominated decoys through the combined use of choice and judgment methods. A second goal was to explore the link between decoy effects in judgment and in choice. The judgment method of studying decoy effects tests predictions of different cognitive models through the use of different types of judgments 
(Wedell \& Pettibone, 1996). The usefulness of this method, however, depends on the linkage between judgment and choice processes.

\section{Nondominated Decoys}

Figure 1 illustrates the three types of nondominated decoys we examined, along with two types of dominated decoys for comparison purposes. Values for alternatives are represented al ong two dimensions, with alternative $A$ superior to $B$ on dimension 2 but not on dimension 1 . The dotted line represents an equipreference contour that assumes equal weighting of both dimensions. Given that A and B lie on the same contour, each alternative should be chosen $50 \%$ of the time in a pairwise choice task. Shaded regions represent classes of alternatives that are dominated by A or B. Only decoys designed to favor the selection of A are shown.

The asymmetrically dominated range decoy favoring $A\left(R_{A}\right)$ extends therange of values on a single dimension, while being dominated on the other. The inferior decoy favoring $A\left(I_{A}\right)$ is very similar to the range decoy, but it has a higher value on dimension 2 so that it is not strictly dominated by either alternative (Huber \& Puto, 1983). The inclusion of the $\mathrm{I}_{\mathrm{A}}$ decoy increases the selection of $A$, the near-dominating alternative. The compromise decoy favoring $A\left(C_{A}\right)$ may share the same value as the inferior and range decoys on dimension 1 , but is raised closer to the equipreference contour due to a higher value on dimension 2 (Simonson, 1989). Unlike the inferior decoy, $C_{A}$ is far from being dominated. Instead, $C_{A}$ results in $A$ having an intermediate value between the decoy and alternative $B$. In these situations, participants prefer the alternative made to look like a compromise. Finally, the phantom decoy $\left(P_{A}\right)$ is unlike other decoys in that it actually dominates the alternative it favors $(A)$. When asked to choose an alternative from the set, participants are told that the decoy is unavailable. Thus, this decoy is a "phantom" alternative, as it is present in the choice set but cannot be selected. Under these conditions, participants prefer the alternative that is dominated by the decoy (Highhouse, 1996; Pratkanis \& Farquhar, 1992). This result is in stark contrast to the effect of a dominated decoy, in which participants select the alternative that dominates another in the set. ${ }^{1}$

\section{Existing Models for Decoy Effects}

Wedell (1991) described three general models of decoy effects. Value-shift models assert that decoys operate through changing the perceived attractiveness of the dimensional values of the alternatives. These changes have been proposed to occur for different reasons. Huber et al. (1982) proposed a range-frequency explanation (Parducci, 1995) for dominated decoys, in which

\footnotetext{
${ }^{1}$ More generally, a phantom decoy could refer to any decoy that is not available for selection at the time of choice. However, in this article we use the term "phantom decoy" to specifically refer to an unavailable alternative that dominates its targeted alternative, as described by Highhouse (1996).
} 
the presence of the decoy either extends the range on one dimension or changes the stimulus rank. For example, the low value of $R_{A}$ on dimension 1 may increase the attractiveness of the dimension 1 value of $A$ because it now lies closer to the midrange of values and is no longer the lowest ranked in the set. This change increases overall attractiveness.

A second mechanism for shifts in values may be through loss aversion (Tversky \& Kahneman, 1991). Simonson and Tversky (1992) suggested a loss-aversion explanation for the effect of the compromise decoy whereby alternatives are valued relative to a referent point, with perceived losses producing a greater change in value than gains. If including $C_{A}$ causes $A$ to become the referent point, then valuation of $C_{A}$ and $B$ will suffer in comparison. This process would result in selecting $A$, as the compromise between two extreme alternatives. Highhouse (1996) also suggested a loss aversion explanation of the phantom decoy. As the most attractive alternative, the phantom decoy sets a referent point from which the other two alternatives are evaluated. As shown in Fig. 1 , relative to the $P_{A}$ decoy, both of the other alternatives represent a loss on dimension 2. Alternative $A$, however, represents a smaller loss on dimension 2 than $B$. The overall perceived loss for A would be evaluated as less than for $B$, despite the fact that $B$ also represents a gain on dimension 1 . This process would result in the selection of $A$, as has been demonstrated in choice. Several lines of research argue for the plausibility of a loss aversion mechanism in choice (Herne, 1998; Highhouse \& J ohnson, 1996).

Weight-change models ascribe decoy effects to changes in the relative weighting or importance of the dimensions (Ariely \& Wallsten, 1995; Huber et al., 1982). Accordingly, the presence of the decoy causes peopleto giveadditional weight to a particular dimension, increasing preference for another alternative in the set. However, there is little evidence to support the weight-change interpretation. With the typical range extension manipulation (e.g., $\mathrm{R}_{\mathrm{A}}$ in Fig. 1), the weight-change model requires a reduction in weight given to the dimension that has been extended. Contrary to this explanation, several lines of evidence across a variety of tasks suggest that participants increase the weight of the extended dimension (Fisher, 1995; Goldstein, 1990, Mellers \& Cooke, 1994; Wedell, 1998; Wedell \& Pettibone, 1996). Highhouse (1996) has also provided evidence against the weight-change explanation of decoy effects.

Both value-shift and weight-change model s are derived from traditional models of choice that assume the attractiveness of alternatives can be described by dimensional values modified by dimension weights (Anderson, 1981; Edwards \& von Winterfeldt, 1986). Emergent-value 2 models of decoy effects diverge from traditional models because they assert that the weighted dimensional values are supplemented by a different type of valuation process. E mergent values are based on the processing of configural information concerning the relationships among alternatives in a set that can provide additional

2 Prior research (Wedell \& Pettibone, 1996) referred to this construct as the value-added model. Based on suggestions from reviewers to clarify the construct, we now refer to it as the emergentvalue model. 
reasons to make a choice. These additional reasons can be thought of as emergent dimensions that arise from the demands of the task or social situation. One such emergent dimension may be the need to justify a decision to others. For example, dominance is an emergent value that provides a qualitative argument for making a choice. When $R_{A}$ in Fig. 1 is added to set $\{A, B\}$, one can justify the selection of $A$ because it is clearly superior to $R_{A}$ while $B$ is not. Such an argument does not rely on integration of weights and dimensional values.

In a similar vein, Simonson (1989) suggested that decoy effects could be driven by a desire to reduce evaluation anxiety related to selecting an alternative. For example, selection of the nondominating alternative would lead to a higher amount of evaluation anxiety because one would expect to be criticized by one's peers for choosing it. Simonson's results (1989) are suggestive of separable justifiability and evaluation anxiety components of the emergent-value construct. With a dominated decoy, increased justifiabi lity and decreased evaluation anxiety were found for the dominating alternative. However, evaluation anxiety decreased for the alternative targeted by the compromise decoy, but there was no increase in justifiability. This differential pattern of results implied justifiability and evaluation anxiety separately contribute to choice, an implication directly tested in the current studies.

Wedell and Pettibone (1996) provided support for a dual-process model involving both value-shift and emergent-value components. This model may berepresented as follows:

$$
A_{i k}=\sum W_{m} V_{i m k}+J_{i k},
$$

where $A_{i k}$ is the overall attractiveness value of alternative $i$ in contextual set $\mathrm{k}, \mathrm{W}_{\mathrm{m}}$ is the weight given to dimension $\mathrm{m}, \mathrm{V}_{\mathrm{imk}}$ is the context dependent scale value of alternative $i$ on dimension $m$ in context $k$, and $J_{i k}$ is the emergent value of alternativei in context $k$ based on relational properties that increase its justifiability. The value-shift component $\left(V_{\text {imk }}\right)$ was supported by demonstrating that dimensional judgments of value shifted with the decoy placement in ways predicted by range-frequency theory (Wedell \& Pettibone, 1996). Similar rangefrequency value-shift effects haveal so been inferred in multiattributejudgment and pairwise choice (Cooke \& Mellers, 1998; Mellers \& Cooke, 1994; Wedell, 1998). The emergent-value component $\left(J_{i k}\right)$ was supported by the demonstration of increased justifiability for an alternative when it dominated the decoy, as well as by showing that justifiability ratings predicted decoy effects on attractiveness ratings over and above that predicted by dimensional value ratings. Wedell and Pettibone (1996) found no support for a weight-change component $\left(\mathrm{W}_{\mathrm{m}}\right)$ tied to context based on ratings of importance.

Validity of the J udgment Approach

Choices provide a limited output record from which to model the cognitive components of decoy effects. Themulti-judgment approach (Wedell \& Pettibone, 
1996) provides a much richer data base by having participants judge different aspects of alternatives in a choice set. The different judgment tasks can then be related to different cognitive processes and used to test corresponding hypotheses concerning decoy effects.

The validity of inferences from the judgment approach depends on establishing construct validity for the separate judgment tasks. In particular, discriminant validity is important to establish so that it is clear that the different overt judgments do not all correspond to the same covert construct. Wedell and Pettibone (1996) most directly addressed the issue of the discriminant validity of the measures in their Experiment 2, in which the symmetrically dominated decoy $\left(\mathrm{RS}_{\mathrm{A}}\right.$ in Fig. 1 ) was studied. Unlike asymmetrically dominated decoys, symmetrically dominated decoys are dominated by both of the other alternatives in the set. Wedell (1991) showed a lack of significant decoy effects in choice when the range was extended on one dimension but the decoy was symmetrically dominated.

Experiment 2 of Wedell and Pettibone (1996) demonstrated that the null effect in choice could be interpreted as reflecting opposing effects of value-shift and emergent-value processes. Specifically, dimensional values significantly shifted in the direction predicted by range-frequency theory and favored the targeted alternative. However, justifiability ratings significantly favored the nontargeted alternative, reflecting the greater ease with which one could detect the dominance of this alternative. Consistent with the two-process model, opposing effects of dimensional values and justifiability led to a null effect of the decoys on attractiveness ratings. These qualitatively different effects on dimensional valuation, justifiability, and attractiveness ratings supported the discriminate validity of these three measures.

\section{Similarity of J udgment and Choice Processes}

Along similar lines, it is also important to establish the relationship between decoy effects in judgment and in choice. Many researchers have demonstrated that choice and judgment differ in important ways that can sometimes lead to different preference patterns (Tversky, Sattath, \& Slovic, 1988). Indeed, some researchers have provided evidence that the processes underlying information integration may qualitatively differ in judgment and choice (Mellers, Ordonez, \& Birnbaum, 1992).

In light of the potential differences between judgment and choice, it is important to determine the degree of overlap between these two response modes in a given task. It may be that both modes use the same processes but in different proportions. For example, the emergent-value component may be emphasized to a greater extent in choice than in judgment. I f so, similar effects would be observed in judgment and choice when both components favored the same alternative, as with the inferior decoy. However, when emergent-value and value-shift processes do not converge on the same alternative, as with the compromise decoy, then different preference patterns might emerge in judgment and choice. 


\section{J udgment Approach with Nondominated Decoys}

We used a multiple-judgment paradigm similar to that of Wedell and Pettibone (1996) to test models for nondominated decoys, with a few small changes. Most notably, the test for the weight-change hypothesis was dropped because of the lack of supporting evidence (Wedell \& Pettibone, 1996) and concerns about task-order effects. This task was replaced by an evaluation-anxiety judgment task to test the possibility of multiple bases of the emergent-value model, as suggested by Simonson's (1989) work. We also collected choice data in a separate session to provide a basis for comparing judgment and choice.

In attractiveness judgment, participants rated the overall attractiveness of each alternative in the choice set. We predicted attractiveness ratings would reflect the same type of decoy effects found in choice based on prior research (Ariely \& Wallsten, 1995; Simonson, 1989; Wedell \& Pettibone, 1996): an alternative should be more attractive when targeted by the decoy.

In dimensi onal valuejudgment, participants rated the attractiveness of each alternative on each of its dimensions. These judgments tested for subjective changes in the dimensional values as predicted by value-shift models. To support a value-shift model, combined dimensional attractiveness ratings should be higher for an alternative when targeted by the decoy.

In justifiability judgment, participants rated how easily they could justify selecting each alternative to their peers. This task was designed to test the emergent-value model. To support it, justifiability ratings should be higher for an alternative when targeted by the decoy.

Finally, in evaluation-anxiety judgment, participants rated the perceived amount of evaluation anxiety associated with selecting each alternative. These judgments were made relative to being evaluated by others. This task was designed to explore the emergent-value model by seeing if we could replicate the distinction between evaluation anxiety and justifiability found by Simonson (1989). As in those experiments, participants were told to rate how anxious they would be if evaluated by a classroom of their peers for choosing a given alternative.

\section{Data Analysis}

To analyze the judgment data generated by the different tasks, an analysis of variance (ANOVA) procedure determined if specific models were being supported by the data. This analysis was supplemented by comparing the data from the dimensional value rating task to the predictions made by rangefrequency theory. Therelationship between judgment and choice was addressed in several ways. First, the ANOVA approach was used to look for decoy effects in choice. Second, to directly compare judgments and choices, the attractiveness judgments were used to infer choice responses by using the highest rated alternative in each choice set to represent the preferred alternative. A correlational analysis was also conducted to address the relationship between judgment and choice, as well as the discriminate validity of the constructs. 


\section{EXPERIMENT 1}

Experiment 1 focused on the inferior decoy and was run in two sessions. In one session, participants made judgments of the three alternatives in 20 different choice sets on each of the four rating dimensions described above. In another session, participants were simply asked to select the alternative they would choose. We expected to find support for the dual-process model by demonstrating significant decoy effects in the dimensional value task and at least one of the emergent-value tasks.

\section{Method}

\section{Participants and Design}

Participants were undergraduates from the University of South Carolina who were told that this experiment would require a two-session commitment. Of these, 81 completed the judgment tasks and 93 completed the choice task, with 69 completing both types of tasks. Participants received course credit for each session for which they volunteered.

Within-subject variables included context (decoy favoring $A$ or $B$ ), choice domain (20 choice sets), and alternative (A or B). Between-subjects variables included task order (four counterbalanced judgment-task orders), session order (choice or judgment first), and set (decoy favors $A$ in sets 1-10 and B in sets 11-20 or favors $A$ in sets $11-20$ and $B$ in sets 1-10). Choice sets were arbitrarily assigned to each block so that interactions with this variable are not of particular interest. Partici pants were randomly assigned to the between-subjects conditions, and presentation order of choice sets was randomized for each participant in each session.

\section{Materials and Apparatus}

Stimuli were based on the 20 choice sets developed by Wedell and Pettibone (1996) and are shown in Appendix A. Each set contained alternatives from a single type of consumer product (e.g., computers, microwaves) or consumer service (e.g., choosing a restaurant, hiring a mechanic), and each was made up of two alternatives ( $A$ and $B$ ) and an inferior decoy, described on two dimensions (e.g., price, ride quality). The inferior decoy was created by lowering the value of the target on its worse dimension by one half the difference between $A$ and $B$, and by raising the value of the better dimension of the target by $1 / 8$ the difference between $A$ and $B$. This resulted in an alternative that was markedly worse than the target on one dimension, and slightly better than the target on the other. All materials and instructions were presented via microcomputers.

\section{Procedure}

For the judgment session, participants were told that they would encounter many sets composed of three alternatives and would make several types of 
judgments for each set. Participants encountered each choice set once in each of four different blocks of trials, with each block corresponding to a different type of judgment. Each choice set was represented as a $3 \times 2$ matrix, with rows corresponding to alternatives and columns to dimensions. On each trial, the arrangement of the alternatives and dimensions on the screen was randomized. Choice sets were presented on the screen $3 \mathrm{~s}$ prior to presentation of the first rating prompt, and theinformation remained on thescreen until all ratings were made. Attractiveness, justifiability, and dimensional value ratings were made on 9-point scales with 1 labeled "not at all" followed by the label "attractive," or "justifiable," and 9 labeled "very," followed by the relevant dimension label. Evaluation anxiety ratings used a similar 9-point scale, but with 1 corresponding to "not very likely to be criticized" and 9 corresponding to "very likely to be criticized."

In the dimensional value task, participants rated all alternatives on a single dimension at a time. For the other judgment tasks, participants rated the alternative at the top of the screen, followed by the middle and bottom alternatives. A blinking judgment cue was used to alert participants to the relevant information being judged. The same display and choice sets were used for the choice session, with participants simply choosing their preferred alternative. Participants were debriefed following the second session.

\section{Results}

\section{Choice}

Consistent with prior research involving the inferior decoy (Huber \& Puto, 1983), the targeted alternative was selected $58 \%$ of the time compared to $29 \%$ of the time for the nontargeted alternative. The decoy was selected $13 \%$ of the time. A repeated-measures ANOVA conducted on the choice proportions for alternatives $A$ and $B$ in the two contextual conditions reveal ed that the Alternative $x$ Context interaction was significant, $F(1,89)=97.3, p<.001$, indicating that an alternative was chosen more often when it was favored by the decoy. ${ }^{3}$

\section{J udgment}

Separate ANOVAs for each judgment task were used to examine the effects of the contextual manipulation. A decoy effect would ber reflected by a significant crossover interaction between context and al ternative, indicating higher attractiveness, justifiability, mean dimensional value, or lower evaluation anxiety for an alternative when it was favored by the decoy. A Context $x$ Alternative interaction in the attractiveness rating task would replicate standard choicebased inferior decoy effects in a judgment task. This interaction in the other

\footnotetext{
${ }^{3}$ For all ANOVA and t test analyses of choice proportions reported in this article, we conducted parallel analyses using the arcsin transformation of the choice proportions. This was done to transform the data into distributions that conformed more closely to the normal distribution. In all of these analyses, the pattern of statistical significance was the same as found for analyses conducted on the choice proportions.
} 
three tasks would provide support for the respective underlying models. M eans for the different tasks collapsed across session and task order are presented in Fig. 2. For all analyses, no higher order interactions with task or session order were observed.

Attractiveness ratings. Theupper left panel of Fig. 2 shows that participants rated an alternative as more attractive when it was favored by the decoy. The crossover interaction of context with alternative was statistically significant, $F(1,65)=43.8, p<.001$, and is consistent with typical results reported for the inferior decoy in choice (Huber \& Puto, 1983) and in judgment (Ariely \& Wallsten, 1995).

J ustifiability ratings. The upper right panel of Fig. 2 shows that participants rated an alternative as more justifiable when it was favored by the decoy,

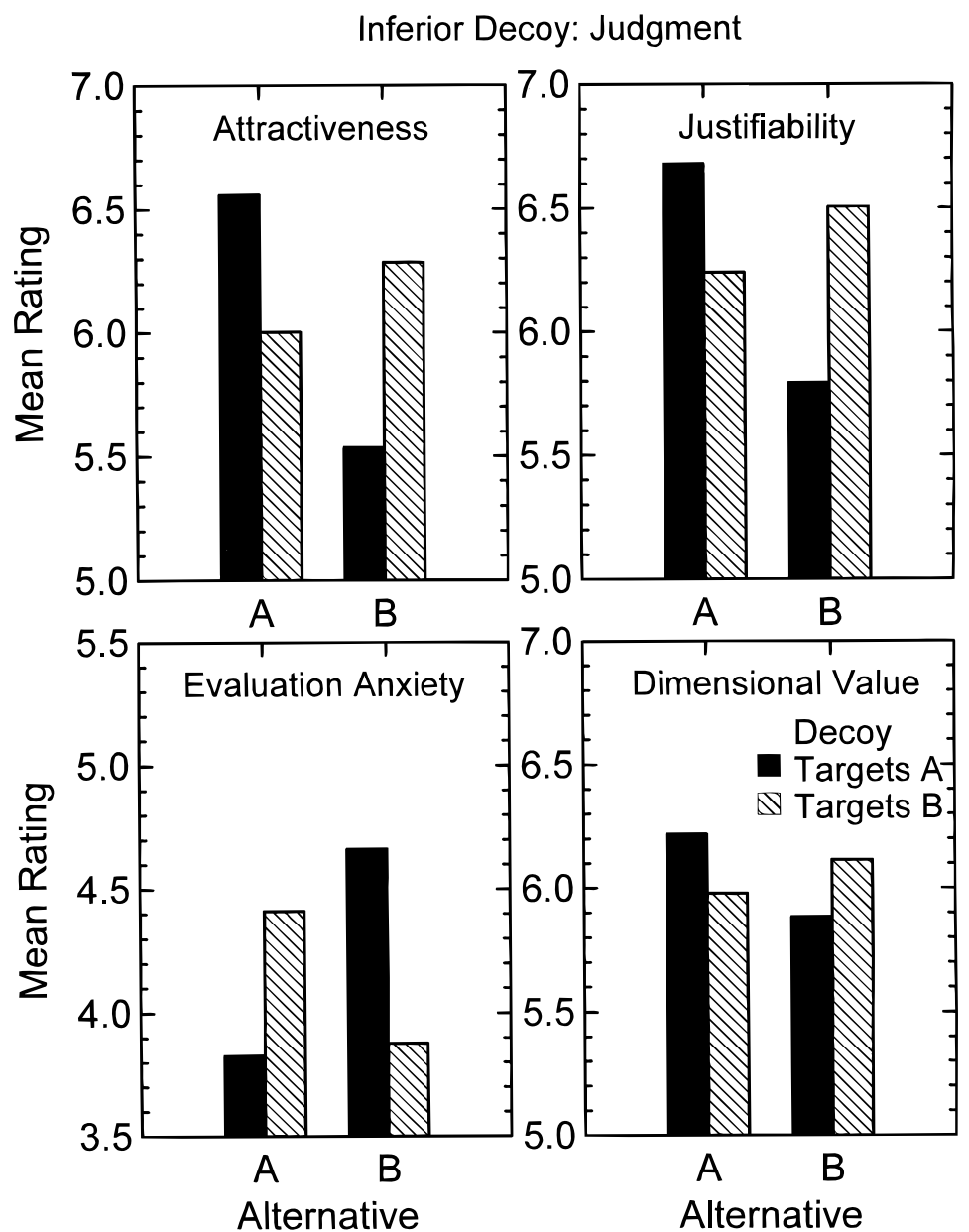

FIG. 2. Mean judgments of alternatives $A$ and $B$ from the attractiveness, justifiability, evaluation anxiety, and dimensional value rating tasks with the inferior decoy (Experiment 1). All interactions of context and alternative are significant. 
consistent with the emergent-value model. This crossover interaction was significant, $F(1,65)=25.2, p<.001$, similar to prior results with asymmetrically dominated decoys (Wedell \& Pettibone, 1996).

Evaluation anxiety ratings. The lower left panel of Fig. 2 shows that an alternative produced less evaluation anxiety when it was favored by the decoy. The crossover interaction was statistically significant, $F(1,65)=35.3, p<.001$, supporting the predictions of the emergent-value model for evaluation anxiety.

Dimensional value ratings. The lower right panel of Fig. 2 shows that the combined dimensional value ratings for an alternative were higher when the decoy favored thealternative. This crossover interaction of context and alternative was significant, $F(1,65)=31.7, p<.001$, supporting the value-shift model. Breaking down these results by dimension revealed two additional significant effects. The Dimension $x$ Alternative interaction was significant, $F(1,65)=$ $605, p<.001$, indicating that on dimension 1 , alternative $B$ was rated higher, but on dimension 2, A was higher. The interaction between dimension and context was also significant, $F(1,65)=214, p<.001$, indicating that the mean rating on dimension 1 was higher when the decoy favored al ternative $A$, but the mean rating on dimension 2 was higher when the decoy favored $B$, supportive of dimensional contrast effects.

\section{Fit of the Range-Frequency Model}

We examined how well range-frequency theory (Parducci, 1995) could predict the strong contextual effects observed by fitting a constrained version of the model to the data from the dimensional value rating task. Allowing both range and frequency to vary with the placement of the decoy resulted in a model with too many free parameters. To compensate for this, range values were held constant across contexts. The equation that we fit to the data was as follows:

$$
\mathrm{J}_{\mathrm{imk}}=\mathrm{wR}_{\mathrm{im}}+(1-\mathrm{w}) \mathrm{F}_{\mathrm{imk}},
$$

where $J_{i m k}$ is the judged value, $R_{i m}$ is the range value, and $F_{i m k}$ is the frequency value of stimulus $i$ on dimension $m$ in context $k$. Frequency values were determined as described by Parducci (1995), with the assumption that the value of $\mathrm{I}_{\mathrm{A}}$ on dimension 2 was within a just noticeable difference of the dimension 2 value for $A$ and thereforeassigned a rank of 2.5 out of 3 . Similarly, thedimension 1 value of $I_{B}$ was assigned a tied rank with the dimension 1 value of $B$. Range values were assumed to be invariant across contexts. The value of the weighting parameter, w, was fit to the data. The fit of this model is displayed in Fig. 3.

Overall, the range-frequency model describes the effect of the inferior decoy very well, with actual values for alternatives $A$ and $B$ shown as open circles and their predicted values shown as solid points. The inferred value of the weighting parameter, $1-\mathrm{w}=.40$, was within the normal range of values found in social judgment research (Wedell, 1994; Wedell, Parducci, \& Geiselman, 1987). All differences in the locations of $A$ and $B$ across contextual conditions are explained by differences in frequency values. 

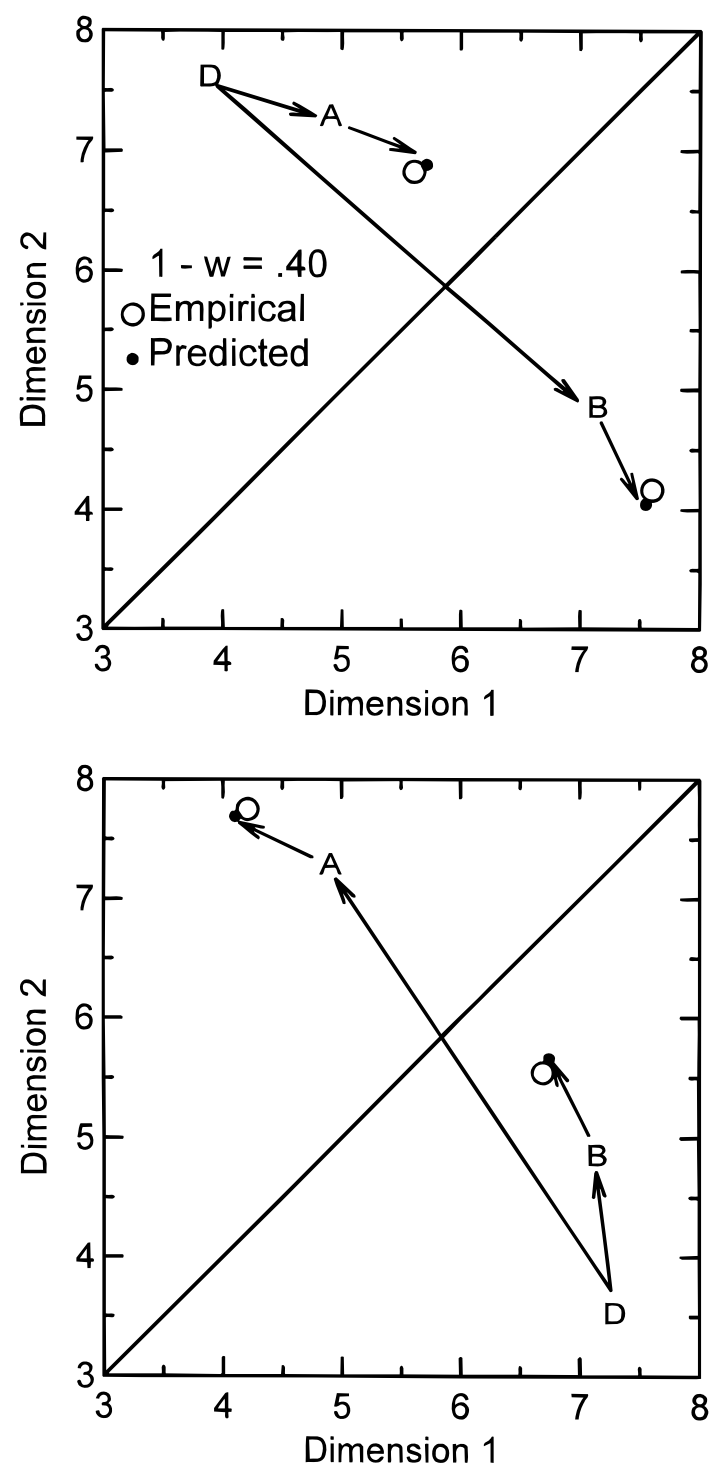

FIG. 3. Comparison of the range-frequency estimates (solid circles) generated from Eq. (2) and mean dimensional value ratings (open circles) with the inferior decoy (D) of Experiment 1 . The arrows from alternatives to data points illustrate contextual shifts from range-frequency processes.

Relationship between Choice and J udgment

To convert attractiveness judgments into preferences, the highest rated alternative on each trial was used as an analog for choice. Cases where a single alternative was not preferred (due to ties) were not included in this analysis. J udgment-based preferences were then compared to the choices for the same stimuli in contingency table form presented in Table 1. Of a total of 1380 possibleresponses from 69 participants, 127 represented ties and were dropped from the analysis. For the $3 \times 3$ contingency table of preferences across choice and judgment, Cohen's Kappa was 0.32, representing moderate agreement 
TABLE 1

Contingency Table of Choices and Preferences Inferred from J udgments (Inferior Decoy)

\begin{tabular}{lcccc}
\hline & \multicolumn{3}{c}{ Choice } \\
\cline { 2 - 5 } J udgment & Target & Other & Decoy & Total \\
\hline Target & 453 & 93 & 68 & 614 \\
& $(36)$ & $(8)$ & $(5)$ & $(49)$ \\
Other & 179 & 250 & 41 & 470 \\
& $(14)$ & $(20)$ & $(3)$ & $(38)$ \\
Decoy & 99 & 21 & $(4)$ & 169 \\
& $(8)$ & $(2)$ & 158 & $(13)$ \\
Total & 731 & $(29)$ & $(13)$ & 1253 \\
& $(58)$ & & \\
\hline
\end{tabular}

Note $\mathrm{N}=69$; frequencies shown along with percentages in parentheses.

across response modes. In Table 1, decoy effects for judgment and choice are represented by the marginal frequencies. The horizontal marginal frequencies represent choice, and the vertical marginal frequencies represent judgment. Decoy effects were strong in both judgment and choice, as demonstrated by the higher preference for the target over the other alternative for both response modes. When directly compared, the inferior decoy effect in judgment was significantly weaker than the effect in choice, with an 18-point reduction from choice to judgment, $\mathrm{t}(68)=-4.03, \mathrm{p}<.01$.

\section{Discussion}

The ANOVA results from Experiment 1 supported both emergent-value and value-shift models of decoy effects with the inferior decoy. This result is similar to that obtained by Wedell and Pettibone (1996) with the asymmetrically dominated decoy, and it demonstrates that the dual-process model can generalize to the inferior decoy. The support found for the emergent-value model is consistent with the possible subjective dominance of the inferior decoy as suggested by others (Ariely \& Wallsten, 1995; Wedell, 1991). Furthermore, the rangefrequency model provided a good account of how values shifted with the presence of the decoy.

Experiment 1 also examined the linkage between decoy effects in choice and judgment. Previous research has argued for the similarity of judgment and choice processes in decoy effects by demonstrating similar effects in both tasks (Ariely \& Wallsten, 1995; Wedell \& Pettibone, 1996). The weaker decoy effect in judgment revealed by the contingency analysis implies that decoy effects in choice and judgment are not identical. The greater sensitivity to decoys in choice may be due to an increased utilization of emergent-value processing. With the inferior decoy, it may be more difficult to justify a choice of any al ternative but thetargeted one, leading people to discount their own judgments 
and choose the alternative that is perceived as most justifiable. We explore this possibility further in Experiment 2.

\section{EXPERIMENT 2}

Experiment 2 focused on the compromise decoy. Because the compromise decoy is unlikely to be subjectively dominated, Experiment 2 should provide a stronger test of the applicability of the dual-process model than did Experiment 1 . The range-frequency model (Parducci, 1995) that forms the basis for the value-shift component predicts opposing dimensional contrast effects that should result in no overall increase in value for the favored alternative. Thus, unlike previous research, we predicted that the combined dimensional values would not favor the target when the compromise decoy is used. Significant results in the dimensional value task favoring the target would be consistent with theuse of a loss-aversion-valuation process rather than a range-frequencybased process. Based on Simonson (1989), we did not expect to find support for changes in justifiability ratings with the compromise decoy. His results suggested that the compromise decoy effect was due solely to an evaluationanxiety-driven mechanism. This pattern would support the divergent validity of justifiability and evaluation anxiety constructs.

\section{Method}

A new set of participants was recruited in the same way as in Experiment 1. Therewere 80 participants in thejudgment session and 80 in choice. Of these, 64 participants completed both sessions. Method and stimuli in Experiment 2 were the same as Experiment 1, except for the use of the compromise decoy in place of the inferior decoy. To create the compromise decoy, we used the same value of the inferior decoy on its worse dimension and substituted the value of the target on its better dimension plus $1 / 2$ the difference between $A$ and $B$. The result is a decoy that makes the targeted alternative a compromise between two extremes yet is still worse in average value than the other two alternatives.

\section{Results}

\section{Choice}

Consistent with prior research involving the compromise decoy (Huber \& Puto, 1983; Simonson, 1989), the targeted alternative was chosen $46 \%$, the nontargeted alternative $32 \%$, and the decoy $22 \%$ of the time. A within-subjects ANOVA conducted on the choice proportions for alternatives $A$ and $B$ in the two contextual conditions revealed that the Alternative $x$ Context interaction was significant, $F(1,76)=20.1, p<.001$, indicating that an alternative was chosen more often when it was favored by the decoy. A significant three-way interaction of alternative, context, and session order, $F(1,76)=5.0, p<.05$, indicated that the magnitude of decoy effects was greater when the choice 
session came after the judgment session. This three-way interaction reflected different magnitudes of decoy effects across session orders and not differences in the direction of the effects.

\section{J udgment}

For all analyses, no higher order interactions with task or session order were observed.

Attractiveness ratings. Theupper left panel of Fig. 4 shows that participants rated an alternative as more attractive when favored by the decoy. This crossover interaction was statistically significant, $F(1,64)=22.4, p<.001$, replicating previous studies that demonstrate decoy effects with the compromise decoy in choice (Simonson, 1989).

J ustifiability ratings. The upper right panel of Fig. 4 shows that participants rated an alternative as more justifiable when favored by the decoy. This

Compromise Decoy: Judgment
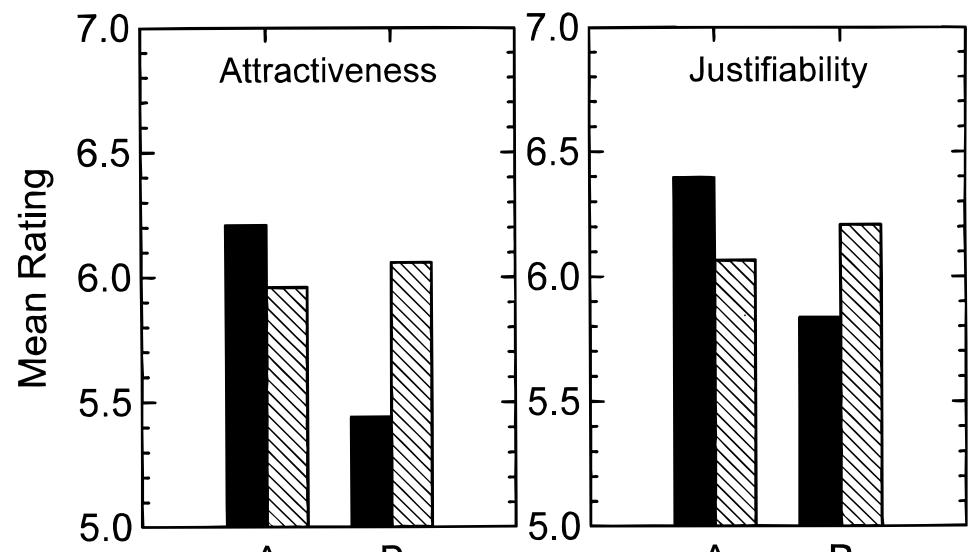

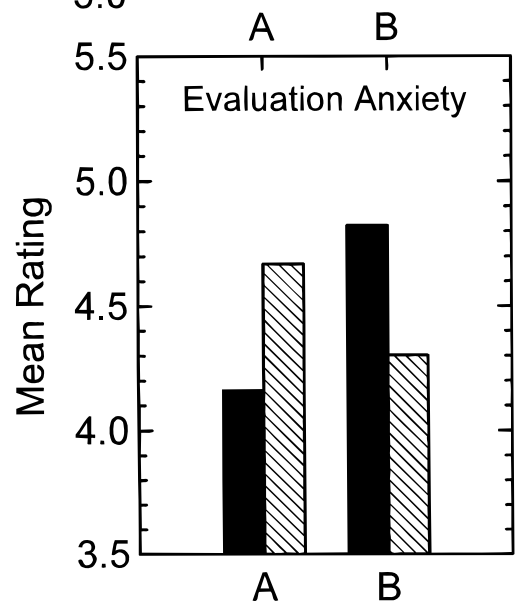

Alternative

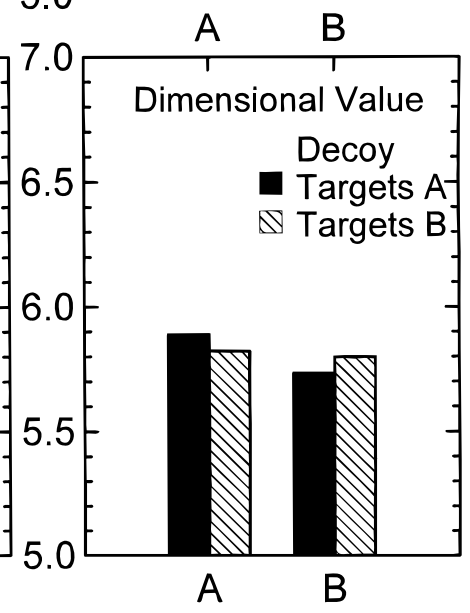

Alternative

FIG. 4. Mean judgments of alternatives $A$ and $B$ from the attractiveness, justifiability, evaluation anxiety, and dimensional value rating tasks with the compromise decoy (Experiment 2). All interactions between alternative and context are significant except for the dimensional value task. 
crossover interaction was significant, $\mathrm{F}(1,64)=15.2, \mathrm{p}<.001$, supporting the emergent-value model and consistent with prior work on asymmetrically dominated decoys (Wedell \& Pettibone, 1996). The interaction, however, is not consistent with results of Simonson (1989), in which no effect of justifiability was found with the compromise decoy.

Evaluation anxiety ratings. The lower left panel of Fig. 4 shows that participants rated an alternativeas producing less evaluation anxiety when favored by the decoy. The crossover interaction of context and alternative was statistically significant, $F(1,64)=20.6, p<.001$, supporting the predictions of the emergent-value model for evaluation anxiety while being consistent with previous work with the compromise decoy (Simonson, 1989).

Dimensional value ratings. Consistent with range-frequency theory, the results shown in the lower right panel of Fig. 4 reflect no significant Alternative $x$ Context interaction, indicating that al ternatives received the same mean dimensional value in both contexts. This result provides no support for the value-shift model with the compromise decoy. The significant Dimension $x$ Context interaction, $F(1,64)=324, p<.001$, did indicate that context effects occurred with the compromise decoy, but offset each other when combined.

\section{Fit of the Range-Frequency Model}

As in Experiment 1, we fit the range-frequency model to the results from the dimensional value rating task with the compromise decoy. Range parameters were again held constant across contexts so that the effects of the decoy were due to frequency values [Eq. (2)]. Unlike Experiment 1, the frequency value of the compromise decoy was not considered tied with any other alternatives on a given dimension. This means that although range-frequency effects were predicted, they would not move the alternatives far from the original preference contour.

The fit of the range-frequency model is shown in Fig. 5, with open circles representing actual results and filled points representing the predicted values. The predictions made by the range-frequency model were very similar to the actual ratings of $A$ and $B$ and were achieved with a best-fit weighting value of $1-\mathrm{w}=.36$. These results were in line with the prediction that conflicting dimensional contrast effects would occur. If a preference contour were drawn through the values of $A$ and $B$, the decoy would simply push the favored alternative along the contour and not above it. Thus, the fit of the rangefrequency model provides an explanation for the lack of support for the valueshift model in the ANOVA section.

\section{Relationship between Choice and J udgment}

Table 2 presents a contingency table comparing actual choices and those inferred from judgment. Of the 1200 possible observations, 84 (7\%) of the total observations were counted as ties and dropped from the analysis. Cohen's 

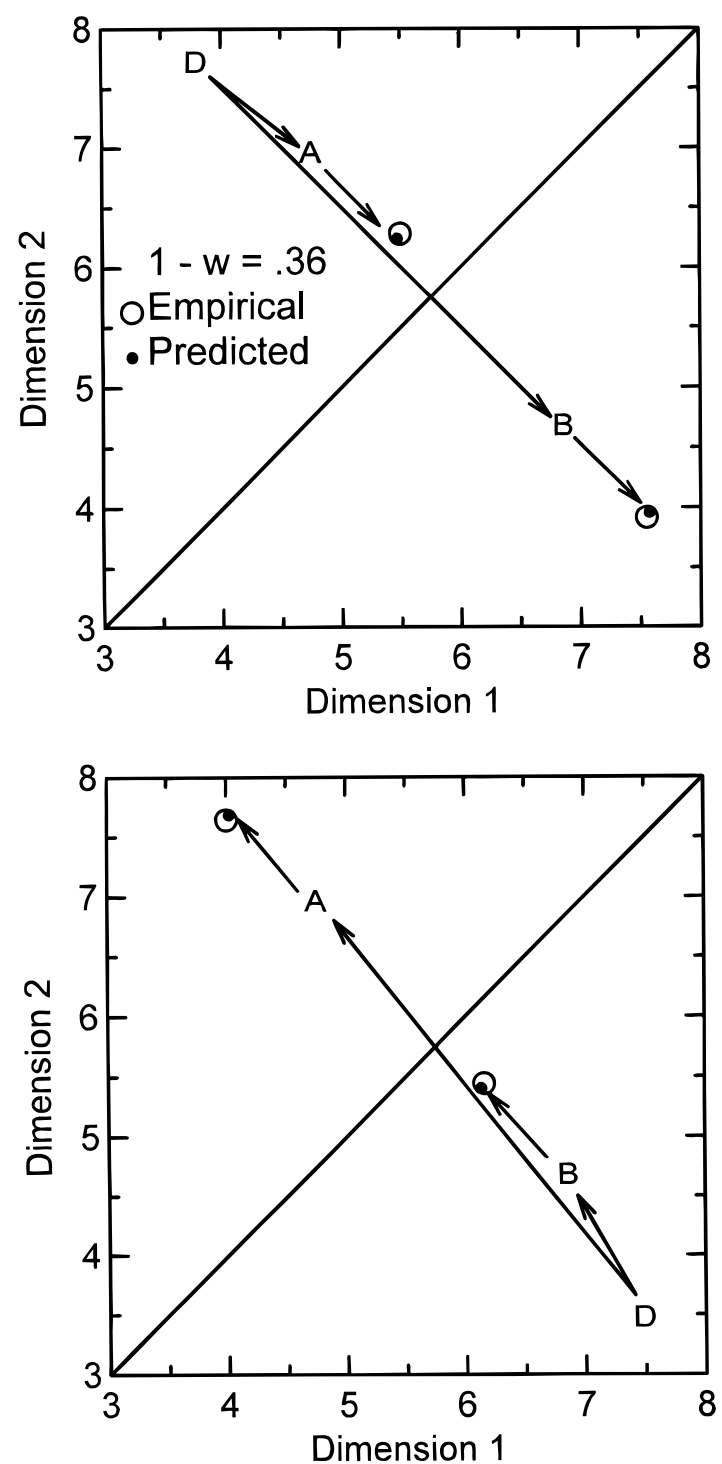

FIG. 5. Comparison of the range-frequency estimates (solid circles) generated from Eq. (2) and mean dimensional value ratings (open circles) with the compromise decoy (D) of Experiment 2. The arrows from alternatives to data points illustrate contextual shifts from range frequency processes.

kappa demonstrated moderate agreement, kappa $=.31$. Examination of the marginal proportions indicates that although decoy effects were found for choice, they were not found for choices inferred from judgment (the 2-point shift was not significant by a t test, $\mathrm{p}>.05$ ). This analysis contradicts the analysis of mean judgments, which demonstrated strong decoy effects on attractiveness ratings. To explore this discrepancy, inferred preferences were broken down further by ranks. As shown in Table 3, mean ratings of the target and other alternativediffered little when they were ranked first or second. H owever, the targeted alternative was rated higher in attractiveness when ranked last, 
TABLE 2

Contingency Table of Choices and Preferences Inferred from J udgments (Compromise Decoy)

\begin{tabular}{lcccc}
\hline & \multicolumn{3}{c}{ Choice } \\
\cline { 2 - 5 } J udgment & Target & Other & Decoy & Total \\
\hline Target & 261 & 98 & 79 & 438 \\
& $(23)$ & $(9)$ & $(7)$ & $(39)$ \\
Other & 132 & 233 & 45 & $(4)$ \\
& $(12)$ & $(21)$ & 123 & $(37)$ \\
Decoy & 106 & 39 & $(11)$ & 268 \\
& $(10)$ & $(4)$ & 247 & 1116 \\
Total & 499 & 370 & $(22)$ & \\
& $(45)$ & $(33)$ & $24)$ \\
\hline
\end{tabular}

Note $\mathrm{N}=60$; frequencies shown along with percentages in parentheses.

contributing to the difference in attractiveness ratings not found in the contingency analysis. A further contribution to the discrepancy between mean judgments and choice inferred from judgment is the large number of observations in which the other alternative is ranked last (369) versus the number of times the target is ranked last (86). Based on the rank order breakdown in Table 3, it would appear that the compromise decoy effect in judgment is not driven by a strong liking for the targeted alternative, but rather by a strong dislike for the other alternative.

\section{Discussion}

The large decoy effects found in both judgment and choice were consistent with previous work with the compromisedecoy (Huber \& Puto, 1983; Simonson, 1989). The contingency table analysis, however, pointed out important differences between judgment and choice. Choices inferred from judgment were similar to the predictions made by the value-shift model, showing no overall preference for the favored alternative. Actual choices were similar to predictions made by the emergent-value model. One possibility for the difference between actual choices and those inferred from judgments observed here is that emergent-value processes were given more weight in choice than in judgment.

TABLE 3

Mean Attractiveness Ratings Broken Down by Rank (Experiment 2, Compromise Decoy)

\begin{tabular}{|c|c|c|c|c|c|c|c|c|}
\hline \multirow[b]{2}{*}{ Alternative } & \multicolumn{2}{|c|}{ Ranked first } & \multicolumn{2}{|c|}{ Ranked second } & \multicolumn{2}{|c|}{ Ranked third } & \multicolumn{2}{|c|}{ Totals } \\
\hline & $\mathrm{N}$ & Mean & $\mathrm{N}$ & Mean & $\mathrm{N}$ & Mean & $\mathrm{N}$ & Mean \\
\hline Target & 327 & 7.67 & 512 & 5.72 & 86 & 4.32 & 925 & 6.27 \\
\hline Other & 356 & 7.65 & 200 & 5.79 & 369 & 3.79 & 925 & 5.71 \\
\hline
\end{tabular}

Note Table includes only those cases where there were no ties among the three alternatives. 
Although a similar trend was suggested in Experiment 1 by the differences in effect size, both value-shift and emergent-value models predicted inferior decoy effects. Therefore, onewould expect to find similar results across response mode, even if people were using different processes. With the compromise decoy, however, only the emergent-value model predicted decoy effects. When making judgments, it appears that participants may have relied more on a value-shift process to determine their ranking of attractiveness. When making choices, the negative properties of the other alternative may have become more salient and led to the selection of the target. The larger effect for the inferior decoy than for the compromise decoy is consistent with the former being driven by both value-shift and emergent-value processes, but the latter being driven by only the emergent-value process.

The range-frequency model correctly predicted the opposing dimensional contrast effects that occurred in the dimensional value task. Because the contrast effects on the dimensions go in opposite directions, no shift in the overall value for an alternative occurs. This result contrasts sharply with results we have reported with inferior and asymmetrically dominated decoys (Wedell \& Pettibone, 1996) in which ANOVA results provided support for the value-shift model. It also argues against a value-shift loss aversion model of the compromise decoy. Finally, the parallel effects found for justifiability and evaluation anxiety ratings do not replicate the pattern found by Simonson (1989), suggesting that these two constructs are not separable.

\section{EXPERIMENT 3}

Experiment 3 focused on the phantom decoy and used the same method as the previous two experiments. As with the compromise decoy, range-frequency theory does not predict the observed decoy effect. Given the position of the $\mathrm{P}_{\mathrm{A}}$ decoy shown in Fig. 1, the range is extended on dimension 2 but not on dimension 1 . Range-frequency theory predicts that this range manipulation should make $B$ preferred to $A$ and thus work against the preference found for target A in choice(Highhouse, 1996). A value-shift implementation of theloss aversion model, however, would predict A to have the higher overall dimensional value. Support for this model would be shown by higher mean dimensional value ratings for the dominated alternative.

\section{Method}

A new set of participants was sampled in the same way as in Experiments 1 and 2 . There were 80 participants in the judgment session, 83 in choice, and 70 completing both sessions. Design features and stimuli of Experiment 3 were similar to those of Experiments 1 and 2, except for the use of the phantom decoy and how stimuli wereassigned to the set variable. For a given participant, half of the choice sets contained phantom decoys and the other half contained three equally attractive alternatives. We did this so participants could not simply assume that only two of the alternatives on a given trial would be 
available. The set variable was expanded to four levels to account for this additional blocking. Results from choice sets containing the three equally attractive alternatives were not analyzed.

To create the phantom decoy, we used the value from the best dimension of the compromise decoy and the value from the worst dimension of the target. This decoy dominated only thetargeted alternative. When participants received a choice set containing a phantom decoy, after the presentation delay, they were told that one of the al ternatives was unavailable due to unforeseen circumstances. The label for the unavailable alternative was made to blink on screen. Participants were then told to rate all alternatives, keeping in mind that one of the alternatives was unavailable. Presentation of a phantom alternative in the choice session was similar, except that participants were told to select from the two available alternatives and were unable to select the phantom decoy.

\section{Results}

\section{Choice}

The targeted alternative was preferred to the other alternative $57 \%$ to $43 \%$ of the time, a difference equivalent to that found for the compromise decoy. Because the decoy alternative could not be chosen, the ANOVA on choice was conducted only on alternative $A$. The main effect of context was significant, $F(1,75)=15.2, p<.001$, indicating that $A$ was selected more often when it was targeted by the decoy and replicating previous work with the phantom decoy (Highhouse, 1996).

\section{J udgment}

For all analyses, no higher order interactions were observed with task or session order.

Attractiveness ratings. The upper left panel of Fig. 6 shows that, unlike the previous two experiments and the choice results from the current experiment, attractiveness ratings did not differ significantly across al ternativeand context, $F(1,48)<1$. The absence of an Alternative $x$ Context interaction indicates that the phantom decoy had little effect on attractiveness ratings.

J ustifiability ratings. The upper right panel of $\mathrm{Fig} .6$ shows that there was no significant Context x Alternative interaction, $F(1,48)<1$, indicating that justifiability for an alternative also did not differ across context. These results fail to support the justifiability component of the emergent-value model with the phantom decoy.

Evaluation anxiety ratings. The lower left panel of Fig. 6 plots ratings of evaluation anxiety associated with choosing an alternative. Although the Context $x$ Alternative interaction is in the predicted direction, with an alternative rated as producing less evaluation anxiety when dominated by the decoy, it was not significant, $F(1,48)=1.2$. The lack of a significant Context $x$ 


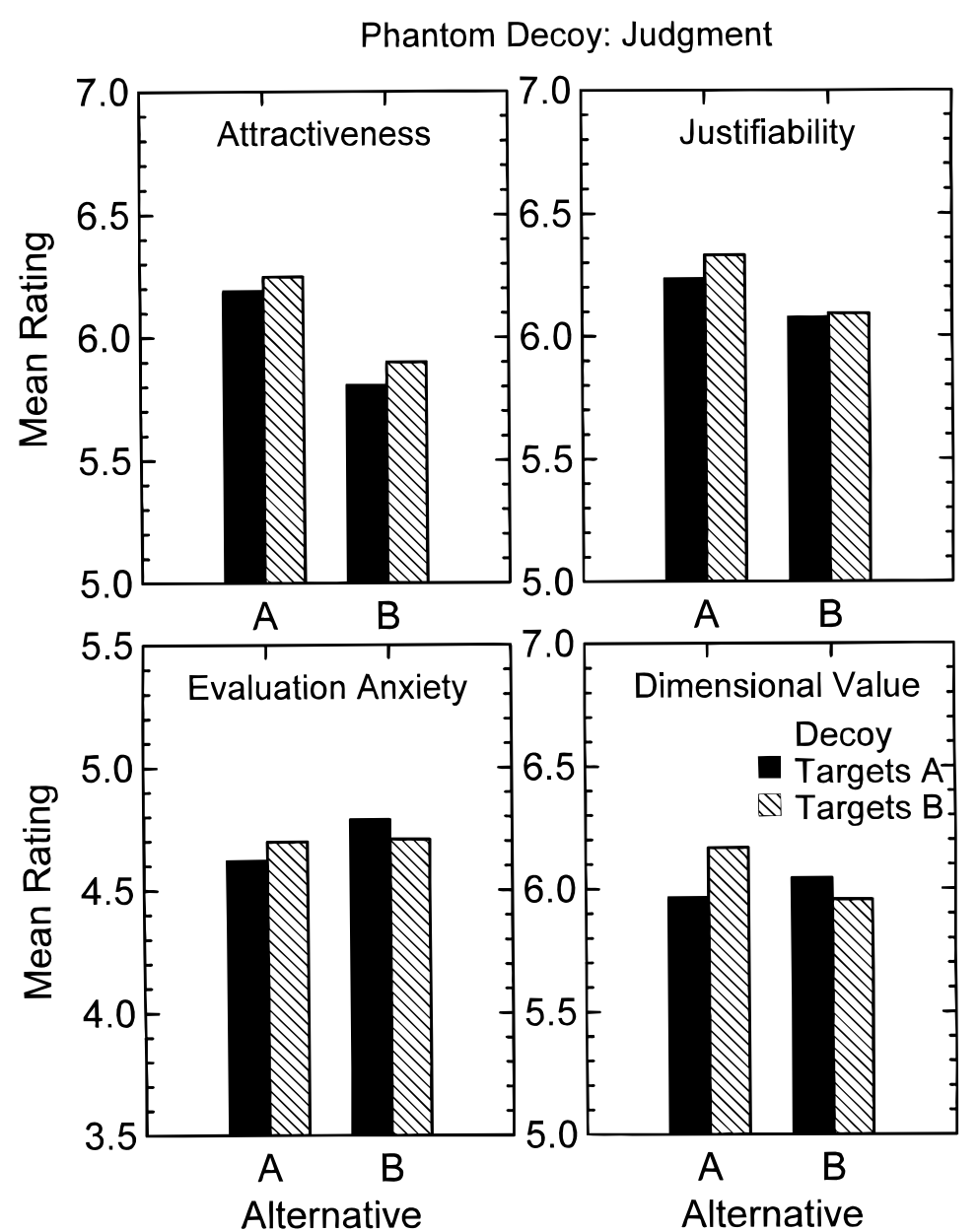

FIG. 6. Mean judgments of alternatives $A$ and $B$ from the attractiveness, justifiability, evaluation anxiety, and dimensional value rating tasks with the phantom decoy (Experiment 3). The interaction between alternative and context is only significant in the dimensional value task.

Alternative interaction fails to support an evaluation anxiety component of the emergent-value model.

Dimensional valueratings. As shown in the lower right panel of Fig. 6, the mean dimensional values for alternatives were lower when dominated by the decoy. The Context $x$ Alternative interaction was significant, $F(1,48)=4.3$, $\mathrm{p}<.05$, supporting the results predicted by range-frequency theory. Note that the direction of this effect operates against the typical phantom decoy effect found in choice.

Relationship between J udgment and Choice

As with the previous experiments, judgments were converted to preferences and compared to choices for the same choice sets. Because participants were 
TABLE 4

Contingency Table of Choices and Preferences Inferred from J udgments (Phantom Decoy)

\begin{tabular}{lccc}
\hline & \multicolumn{3}{c}{ Choice } \\
\cline { 2 - 4 } J udgment & Target & Other & Total \\
\hline Target & 255 & 83 & 338 \\
& $(41)$ & $(13)$ & $(54)$ \\
Other & 99 & 183 & 282 \\
& $(16)$ & $(30)$ & $(45)$ \\
Total & 354 & 266 & 620 \\
& $(57)$ & $(43)$ & \\
\hline
\end{tabular}

Note $N=70$; frequencies shown along with percentages in parentheses.

unable to select the decoy in choice, we constructed a $2 \times 2$ contingency table including only thetarget and other alternative across both judgment and choice. For those participants who rated the decoy the highest, their second highest preference was used. Each of 70 participants who completed both choice and judgment sessions received 10 choice sets that contained a phantom decoy, for a total of 700 possible responses. Of these, 80 responses (11\%) reflected ties in the judgment session and were dropped. These data are presented in Table 4, and they show moderate agreement across response modes, kappa $=.41$.

The marginal frequencies indicate that the targeted alternative was preferred by a similar margin in both response modes. Although this replicates the effect found in the ANOVA analysis for choice, it is not consistent with the lack of decoy effects found in attractiveness ratings. This difference can be understood by looking at therank information provided in Table 5. Participants rarely ranked the targeted alternative highest because the decoy dominates it. They ranked the nontargeted alternative highest twice as often as the targeted alternative. However, they ranked the decoy (not shown in Table 5) highest more often than either of these (44\% of judgments). Because the decoy cannot be chosen in these cases, preference falls to the alternative ranked second. Here the targeted al ternative enjoys a strong advantage over the other alternative. Thus, when inferred choices exclude choice of the decoy, the strong tendency to rank the target second leads to the phantom decoy effect. This

\section{TABLE 5}

Mean Attractiveness Ratings Broken Down by Rank (Experiment 3, Phantom Decoy)

\begin{tabular}{|c|c|c|c|c|c|c|c|c|}
\hline \multirow[b]{2}{*}{ Alternative } & \multicolumn{2}{|c|}{ Ranked first } & \multicolumn{2}{|c|}{ Ranked second } & \multicolumn{2}{|c|}{ Ranked third } & \multicolumn{2}{|c|}{ Totals } \\
\hline & $\mathrm{N}$ & Mean & $\mathrm{N}$ & Mean & $\mathrm{N}$ & Mean & $\mathrm{N}$ & Mean \\
\hline Target & 71 & 7.78 & 261 & 6.29 & 136 & 4.59 & 468 & 6.02 \\
\hline Other & 140 & 7.75 & 83 & 6.06 & 245 & 4.45 & 468 & 5.72 \\
\hline
\end{tabular}


advantage is not found in the mean ratings because it is balanced out by the greater tendency to rank the nontargeted alternative highest.

\section{Discussion}

Results from the choice task replicated the phantom decoy effect previously observed (Highhouse, 1996). The most remarkable finding from Experiment 3 , however, was the failure to demonstrate phantom decoy effects with judgment, even though our experiments had high power to detect the predicted interaction effect. ${ }^{4}$ The lack of an effect on attractiveness judgments means that while choice preferences may be altered by the presence of the phantom decoy, judgment preferences are not. The phantom decoy does not make the favored alternative either more justifiable or less anxiety provoking, so that emergent-value models do not appear to explain its selection. Consistent with range-frequency theory, the phantom decoy makes the targeted alternative's combined dimensional values less attractive, providing no route for the valueshift component to produce the decoy effect in choice. ${ }^{5}$

A similarity-substitution hypothesis (Tversky, 1972) provides one explanation of the discrepancy between judgment and choice behavior. According to this model, the phantom decoy effect is a choice-based effect that results from substituting a similar alternative when the preferred alternative (the decoy) is not available. Based on judgments al one, thetarget and the other alternative are of roughly equal attractiveness. Because one cannot select the alternative actually preferred in choice, the default becomes the next most similar alternative, i.e., the target. This can save effort by preventing one from having to reevaluate the reduced set of alternatives. Naturally, the post hoc nature of this explanation suggests that it must be tested more carefully. Furthermore, other models, such as loss aversion (Highhouse, 1996), might explain these results if one assumes that they operate in choice but not in judgment.

\footnotetext{
${ }^{4}$ We investigated whether the failure to reject the null hypotheses in the three judgment tasks was due to a lack of power. Because observed decoy effects in choice were similar across the phantom and compromise decoys (a 14\% shift in each), effect sizes from the judgment tasks with the compromise decoy were used to estimate corresponding effect sizes for the phantom decoy. The error term was based on the mean-squared error from the phantom decoy (Experiment 3). The power to detect effects comparable to those observed in Experiment 2 was high for both attractiveness and evaluation anxiety judgments (power of .96 and .92, respectively). Power was moderate for justifiability judgments, .76. These results indicate that Experiment 3 had adequate power to detect phantom decoy effects in these three tasks.

${ }^{5}$ To investigate the possibility that the absence of decoy effects in judgment was due to the participants having to rate the phantom decoy, we conducted an additional experiment with only a judgment session in which the decoy information was not overtly rated. Those results replicated the judgment results from Experiment 3 for all tasks except for dimensional value judgment in which there were no significant effects of decoy. These results indicate that even when explicit ratings were only made for the target and other alternative, there is still no preference for the target reflected in judgments of attractiveness, justifiability, or evaluation anxiety.
} 


\section{Correlational Analysis}

To further explore the relationships between our constructs in judgment and between judgment and choice, we created contextually sensitive interaction scores for each rating task from each experiment. Appendix B displays the correlations among these scores. We highlight two results. First, correlations between the evaluation anxiety and justifiability tasks were high, ranging from .52 to .67. This result provides little evidence for the divergent validity for these components of the emergent-value model. Second, correlations between individual differences in decoy effects across choice and attractivenessjudgment tasks were moderately high, averaging $r=.50$ for Experiments 1 and 2. In Experiment 3, where large differences were found between judgment and choice, this correlation was lower, $r=.29$.

\section{GENERAL DISCUSSION}

We have extended research on decoy effects by examining effects of compromise, inferior, and phantom decoys across two response modes. We bel ieve the results from the combination of these two approaches provide insights into the processes underlying effects of nondominated decoys.

\section{Similarity between J udgment and Choice Processes}

Experiments 1 and 2 demonstrated that the processes underlying decoy effects in choice and judgment are related, but not identical. First, similar effects of both compromise and inferior decoys were demonstrated in choice and judgment. Second, choices and inferred choices derived from judgments were moderately related, as demonstrated by kappa values in the .3- .4 range. Third, individual differences in judgment were moderately correlated with those in choice. These results provide direct evidence of what until this point has been assumed, namely, that decoy effects in judgment reflect decoy effects in choice(Ariely \& Wallsten, 1995; Dhar \& Glazer 1996; Wedell \& Pettibone, 1996).

Experiments 2 and 3 also demonstrated that judgment and choice processes may differ in important ways. Although overall attractiveness ratings reflected significant effects of the compromise decoy in Experiment 2, these effects disappeared when the ratings were converted to inferred choices. This difference between choice and inferred choice can be explained by proposing that participants rely more on emergent-value processing in choice than in judgment.

Results for the phantom decoy provide additional evidence supporting a distinction between judgment and choice. In Experiment 3, decoy effects were absent from the judgment record, except in the case of dimensional value ratings which operated against the selection of the target. Emergent-value processing was not supported in the judgment record, but the significant dimensional value ratings indicated that value-shift processing was still occurring. Choice, however, demonstrated strong phantom decoy effects. This difference between choice and judgment is consistent with a similarity-substitution model 
that assumes phantom decoy effects may arise in choice due to a strategy of selecting the alternative most similar to the preferred alternative.

\section{Dual-Process Model}

To what extent are the dual processes of the value-shift and emergent-value models (Wedell \& Pettibone, 1996) necessary to explain the effects of nondominated decoys? In the present research, both processes appeared to operate in favor of the targeted alternative only for the inferior decoy. Results suggested no role for a value-shift component in determining the effects of the compromise decoy. The emergent-valuemodel appeared to hel p explain effects of thecompromise decoy, but not the phantom decoy.

J udgments of dominated decoys have provided the strongest support for the value-shift model (Wedell \& Pettibone, 1996). The current set of experiments suggests that as the decoy is positioned farther away from being dominated, the value-shift model is less likely to explain decoy effects. Instead, emergentvalue processes that depend on relationships among alternatives in the set take on greater significance. Our range-frequency analyses supported this conclusion, demonstrating that dimensional contrast effects cancel out for the compromise decoy so that there is no overall increase in attractiveness. If participants were basing their decision on dimensional contrast effects proposed by the value-shift model, then there should have been no decoy effects with the compromise decoy. Thus, value-shift processes could not explain the effect of the compromise decoy, and indeed they predicted the opposite effect than was found with the phantom decoy in choice. The different pattern of effects for combined dimensional attractiveness ratings versus that for global attractiveness ratings also provides support for the discriminant validity of these constructs.

To what extent doweneed to break down theemergent-valuemodel down into justifiability and evaluation anxiety components? Both components showed parallel effects for both the compromise and inferior decoys. Thus, we were unable to replicate the differential effects of the compromise decoy on these as reported by Simonson (1989). Correlations between the two components were consistently high, failing to establish divergent validity. In summary, our experiments provide little support for the separation of these two emergent-value constructs.

These conclusions must be tempered by the fact that our measurement of evaluation anxiety may not have been as strong as that used by others. In contrast toSimonson's (1989) work, participants never actually had to beevaluated by others. Thus, the lack of effects observed in our experiments may have been due to this difference rather than because they are not distinct constructs. To address this issue, future experiments should include a peer discussion session after all judgments are made in which participants will discuss their actions and be evaluated. Along similar lines, directly manipulating the accountability of the participants would provide a more direct test of the emergent-value hypothesis as a whole. 
Although value-shift processes are a viable explanation for many decoy effects, only emergent-value processes have proven capable of predicting significant decoy effects across inferior, compromise, and asymmetrically dominated decoys. F urthermore, the data presented heresuggest that a different emphasis may be placed on emergent-value and value-shift processing in judgment and choice. The present results bolster the case we have made in our previous work that models of the choice process need to take into account aspects of the choice set that go beyond dimensional weights and values. The observable relationships between alternatives, the ability to justify a choice, or the goal to avoid decision based anxiety may be as important as the actual underlying values or weights. Incorporation of these emergent-value processes provides an explanation for contextual effects where there are no underlying shifts in values or weights.

\section{APPENDIX A}

Choice Sets Used in Experiments 1-3

\section{Domain/dimension}

\section{Computers}

1. Processing Speed (Mhz)

2. Size of hard drive (MB)

2. Restaurants

1. Price of meal for two (\$)

2. Wait to be served (min.)

3. Plane tickets

1. Cost of ticket (\$)

2. Length of layover (min.)

4. Mechanics

1. Warranty Length (days)

2. Experience (years)

5. CD Players

1. Price $(\$)$

2. Number of disks

6. Apartments

1. Rent per month (\$)

2. Distance (min.)

\section{Cars}

1. Miles per gallon

2. Number of safety features

8. Boats

1. Number of passengers

2. Speed (Knots per hour)

9. J ob offers

1. Days of sick leave

2. Number of paid holidays

10. Houses

1. Price (thousands of \$)

2. Square footage

$\begin{array}{llllllll}A & B & I_{A} & I_{B} & C_{A} & C_{B} & P_{A} & P_{B}\end{array}$

\begin{tabular}{rrrrrrrr}
40 & 66 & 27 & 69 & 27 & 79 & 40 & 79 \\
420 & 300 & 432 & 240 & 480 & 240 & 480 & 300 \\
44 & 36 & 48 & 35 & 48 & 32 & 44 & 32 \\
20 & 34 & 18 & 41 & 14 & 41 & 14 & 34 \\
& & & & & & & \\
400 & 330 & 435 & 323 & 435 & 295 & 400 & 295 \\
60 & 150 & 51 & 195 & 15 & 195 & 15 & 150 \\
& & & & & & & \\
30 & 60 & 15 & 63 & 15 & 75 & 30 & 75 \\
14 & 7 & 15 & 4 & 17 & 4 & 17 & 7 \\
& & & & & & & \\
250 & 175 & 288 & 167 & 288 & 138 & 250 & 138 \\
10 & 4 & 11 & 1 & 13 & 1 & 13 & 4 \\
& & & & & & & \\
200 & 140 & 230 & 134 & 230 & 110 & 200 & 110 \\
10 & 20 & 9 & 25 & 5 & 25 & 5 & 20 \\
& & & & & & & \\
22 & 35 & 16 & 37 & 16 & 41 & 22 & 41 \\
10 & 5 & 11 & 3 & 12 & 3 & 12 & 5 \\
& & & & & & & \\
4 & 20 & 1 & 22 & 1 & 28 & 4 & 28 \\
30 & 10 & 32 & 5 & 40 & 5 & 40 & 10 \\
& & & & & & & \\
6 & 10 & 4 & 11 & 4 & 12 & 6 & 12 \\
16 & 12 & 17 & 10 & 18 & 10 & 18 & 12 \\
75 & 40 & 92.5 & 36.5 & 92.5 & 22.5 & 75 & 22.5 \\
1500 & 1100 & 1540 & 900 & 1700 & 900 & 1700 & 1100 \\
\hline & & & & & & &
\end{tabular}




\section{APPENDIX A-Continued}

\section{Domain/dimension}

11. Electric Keyboards

1. Tone quality (1-100)

2. Number of features

12. Mini-LCD TVs

1. Price (\$)

2. Percent distortion

13. Preschools

1. Children per class

2. Teacher experience (years)

14. Microwaves

1. Warranty (months)

2. Cooking power (Watts)

15. Parking spaces

1. Price per month (\$)

2. Distance from job (blocks)

16. Video cameras

1. Weight (pounds)

2. Number of features

17. Beer (24 pack)

1. Price $(\$)$

2. Quality (1 to 100 )

18. Cars

1. Ride quality (1 to 100 )

2. Miles per gallon

19. Restaurants

1. Distance from home ( $\mathrm{min}$.)

2. Quality/star (1 to 5)

20. TV sets (19 inch)

1. Percent distortion

2. Average life span (years)

\begin{tabular}{llllllll}
$A$ & $B$ & $I_{A}$ & $I_{B}$ & $C_{A}$ & $C_{B}$ & $P_{A}$ & $P_{B}$ \\
\hline
\end{tabular}

$\begin{array}{rrrrrrrr}75 & 85 & 70 & 86 & 70 & 90 & 75 & 90 \\ 15 & 10 & 16 & 8 & 18 & 8 & 18 & 10 \\ & & & & & & & \\ 195 & 100 & 218 & 90 & 218 & 53 & 195 & 53 \\ 2 & 4 & 1.75 & 5 & 1 & 5 & 1 & 4\end{array}$

$\begin{array}{llllclll}12 & 8 & 10 & 7 & 10 & 6 & 12 & 6 \\ 12 & 5 & 12 & 2 & 15 & 2 & 15 & 5\end{array}$

$\begin{array}{rrrrrrrr}8 & 14 & 5 & 15 & 5 & 17 & 8 & 17 \\ 1600 & 900 & 1670 & 550 & 1950 & 550 & 1950 & 900\end{array}$

$\begin{array}{llllllll}50 & 25 & 62 & 22 & 62 & 13 & 50 & 13\end{array}$

$\begin{array}{llllllll}3 & 6 & 2.5 & 7.5 & 1.5 & 7.5 & 1.5 & 6\end{array}$

$\begin{array}{rlrlrlrl}7.0 & 4.0 & 8.5 & 3.7 & 8.5 & 2.5 & 7 & 2.5 \\ 14 & 8 & 15 & 5 & 17 & 5 & 17 & 8\end{array}$

$\begin{array}{llllllll}10 & 8.50 & 10.75 & 8.35 & 10.7 & 7 & 10 & 7\end{array}$

$\begin{array}{llllllll}60 & 50 & 61 & 45 & 65 & 45 & 65 & 50\end{array}$

$\begin{array}{llllllll}65 & 80 & 57 & 82 & 57 & 87 & 65 & 88\end{array}$

$\begin{array}{llllllll}30 & 24 & 31 & 21 & 33 & 21 & 33 & 24\end{array}$

$\begin{array}{llllllll}45 & 15 & 60 & 12 & 60 & 5 & 45 & 5\end{array}$

$\begin{array}{llllllll}4 & 3 & 4.25 & 2 & 4.5 & 2 & 4.5 & 3\end{array}$

$\begin{array}{llllllll}3.5 & 2.5 & 4 & 2 & 4 & 1.5 & 3.5 & 4.5\end{array}$

$\begin{array}{llllllll}4 & 3 & 4.5 & 2 & 5 & 2 & 2.0 & 3\end{array}$

\section{APPENDIX B}

\section{Correlation Matrices of Rating Tasks for the Compromise, Inferior, and Phantom Decoys}

\begin{tabular}{lcccc}
\hline & \multicolumn{4}{c}{ Rating tasks } \\
\cline { 2 - 5 } \multicolumn{1}{c}{ Decoy/rating task } & Attractiveness & J ustifiability & $\begin{array}{c}\text { Evaluational } \\
\text { anxiety }\end{array}$ & $\begin{array}{c}\text { Dimensional } \\
\text { value }\end{array}$ \\
\hline Inferior $(\mathrm{N}=81)$ & & & & \\
J ustifiability & $.63^{*}$ & & & \\
Evaluational anxiety & $-.58^{*}$ & $-.67^{*}$ & & \\
Dimensional value & $.44^{*}$ & $.49^{*}$ & $-.37^{*}$ & $.29 *$ \\
Choice $^{\mathrm{a}}$ & $.55^{*}$ & $.44^{*}$ & $-.41^{*}$ & \\
\hline
\end{tabular}




\section{APPE NDIX B-Continued}

\begin{tabular}{lcccc}
\hline & \multicolumn{4}{c}{ Rating tasks } \\
\cline { 2 - 5 } \multicolumn{1}{c}{ Decoy/rating task } & Attractiveness & J ustifiability & $\begin{array}{c}\text { Evaluational } \\
\text { anxiety }\end{array}$ & $\begin{array}{c}\text { Dimensional } \\
\text { value }\end{array}$ \\
\cline { 2 - 5 } Compromise $(\mathrm{N}=80)$ & & & & \\
J ustifiability & $.49^{*}$ & & & \\
Evaluational anxiety & $-.32^{*}$ & $-.52^{*}$ & & $.24^{*}$ \\
Dimensional value & $.40^{*}$ & $.44^{*}$ & $-.41^{*}$ & \\
Choice & $.45^{\mathrm{a}}$ & $.32^{*}$ & $-.41^{*}$ & \\
Phantom (N = 80) & & & & \\
J ustifiability & $.65^{*}$ & & & $.21^{*}$ \\
Evaluational anxiety & $-.65^{*}$ & $-.67^{*}$ & & \\
Dimensional value & $.67^{*}$ & $.68^{*}$ & $-.45^{*}$ & \\
Choice & $.29 *$ & .14 & -.14 & \\
\hline
\end{tabular}

${ }^{\text {a }}$ Choice correlations are based on a smaller $\mathrm{N}$ due to the fact that not all participants returned to complete both judgment and choice sessions; N was 68, 69, and 70 for inferior, compromise, and phantom decoys respectively.

* Significance at the .05 level or below.

\section{REFERENCES}

Anderson, N. H. (1981). Foundations of information integration theory. New York: Academic Press.

Ariely, D., \& Wallsten, T. S. (1995). Seeking subjective dominance in multidimensional space: An explanation of the asymmetric dominance effect. Organizational Behavior and Human Decision Processes, 63, 223-232.

Cooke, A. D. J ., \& Mellers, B. A. (1998). Multiattribute judgment: Attribute spacing influences single attributes. J ournal of Experimental Psychology: Human Perception and Performance, 24, 496-504.

Dhar, R., \& Glazer, R. (1996). Similarity in context: Cognitive representation and violation of preference and perceptual invariance in consumer choice. Organizational Behavior and Human Decision Processes, 67, 280-293.

Doyle, J . R., O'Connor, D. J ., Reynolds, G. M., \& Bottomley, P. A. (1999). The robustness of the asymmetrically dominated effect: Buying frames, phantom alternatives, and in-store purchases. Psychol ogy \& Marketing, 16, 225-243.

Edwards, W., \& von Winterfeldt, D. (1986). On cognitive illusions and their implications. In H. R. Arkes \& K. R. Hammond (Eds.), J udgment and decision making: An interdisciplinary reader (pp. 642-679). Cambridge: Cambridge Univ. Press.

Fisher, G. W. (1995). Range sensitivity of attribute weights in multiattribute value models. Organizational Behavior and Human Decision Processes, 62, 252-266.

Goldstein, W. M. (1990). J udgments of relative importance in decision making: Global vs local interpretations of subjective weight. Organizational Behavior and Human Decision Processes, 47, 313-336.

Herne, K. (1998). Testing the reference-dependent model: An experiment on asymmetrically dominated reference points. J ournal of Behavioral Decision Making, 11, 181-192.

Highhouse, S. (1996). Context-dependent selection: The effects of decoy and phantom job candidates. Organizational Behavior and Human Decision Processes, 65, 68-76. 
Highhouse, S., \& J ohnson, M. A. (1996). Gain/Loss asymmetry and riskless choice: Loss aversion in choices among job finalists. Organizational Behavior and Human Decision Processes, 68, 225-233.

Huber, J ., Payne, J . W., \& Puto, C. (1982). Adding asymmetrically dominated alternatives: Violations of regularity and the similarity hypothesis. J ournal of Consumer Research, 9, 90-98.

Huber, J ., \& Puto, C. (1983). Market boundaries and product choice: Illustrating attraction and substitution effects. J ournal of Consumer Research, 10, 31- 44.

Mellers, B. A., \& Cooke, A. D. J . (1994). Trade-offs depend on attributerange. J ournal of Experimental Psychology: Human Perception and Performance, 20, 1055- 1067.

Mellers, B. A., Ordonez, L. D., \& Birnbaum, M. H. (1992). A change-of-process theory for contextual effects and preference reversals in risky decision making. Organizational Behavior and Human Decision Processes, 52, 331- 369.

Parducci, A. (1995). Happiness, pleasure, and judgment: Thecontextual theory and its applications. Mahwah, NJ : Erlbaum.

Pratkanis, A. R., \& Farquhar, P. H. (1992). A brief history of research on phantom alternatives: Evidence for seven empirical generalizations about phantoms. Basic and Applied Social Psychology, 13, 103-122.

Simonson, I. (1989). Choice based on reasons: The case of attraction and compromise effects. J ournal of Consumer Research, 16, 158-174.

Simonson, I., \& Tversky, A. (1992). Choice in context: Tradeoff contrast and extremeness aversion. J ournal of Marketing Research, 29, 281-295.

Tversky, A. (1972). Elimination by aspects: A theory of choice. Psychological Review, 79, 281-299.

Tversky, A., \& Kahneman, D. (1991). Loss aversion in riskless choice: A reference-dependent model. Quarterly J ournal of Economics, 106, 1039- 1061.

Tversky, A., Sattath, S., \& Slovic, P. (1988). Contingent weighting in judgment and choice. Psychological Review, 95, 371-384.

Wedell, D. H. (1991). Distinguishing among models of contextually induced preference reversals. J ournal of Experimental Psychology: Learning, Memory, and Cognition, 17, 767-778.

Wedell, D. H. (1994). Contextual contrast in evaluative judgments: Test of pre- versus post-integration models of contrast. J ournal of Personality and Social Psychology, 66, 1007-1019.

Wedell, D. H. (1998). Testing models of trade-off contrast in pairwisechoice. J ournal of Experimental Psychology: Human Perception and Performance, 24, 49-65.

Wedell, D. H., Parducci, A., \& Geiselman, R. E. (1987). A formal analysis of ratings of physical attractiveness: Successive contrast and simultaneous assimilation. J ournal of Experimental Social Psychology, 23, 230-249.

Wedell, D. H., \& Pettibone, J . C. (1996). Using judgments to understand decoy effects in choice. Organizational Behavior and Human Decision Processes, 67, 326- 344.

Received April 21, 1998 